\section{W Mass Measurement at LEP 2}

P. Perez and A. Trabelski

Centre d'Etudes de Saclay, C. E. A., France

LEP, the large $e^{+} e^{-}$collider located at CERN, Geneva, has been operated for the first time at centre of mass energies of 161 and $172 \mathrm{GeV}$ during the year 1996 , starting phase 2 of its run. Such energies are higher than twice the mass of the $\mathrm{W}$ boson, the charged mediator of the weak force. Thus pairs of real $\mathrm{W}$ bosons are created in the reaction, allowing for a precise determination of their intrinsic properties as well as those of their production.

The $\mathrm{W}$ mass is a fundamental parameter of the electroweak theory. Other parameters like the mass of the $\mathrm{Z}$ boson, the neutral partner of the W, (re. the Fermi constant) are already known with a relative uncertainty of $2 \cdot 10^{-5}$ $\left(2 \cdot 10^{-6} \mathrm{resp}.\right)$. In the Born approximation, these parameters are related. This relation is modified when quantum corrections are taken into account, which correspond to the effects of other fundamental particles like quarks, Higg's bosons or even supersymmetric particles in the case where the Standard Model of weak interactions is extended with a boson-fermion symmetry. The biggest calculated effect for these socalled radiative corrections is due to the top-bottom quark mass difference. The top mass has been directly measured at the Fermilab proton-antiproton collider:
$176 \pm 6 \mathrm{GeV} / \mathrm{c}^{2}$. The main remaining unknown comes from the still undiscovered Higg's boson, responsible for the generation of mass. The Higg's boson enters the radiative corrections with a term proportional to the logarithm of its mass. Present measurements constrain the mass of the Standard Model Higgs within an interval $65600 \mathrm{GeV} / \mathrm{c}^{2}$.

At Fermilab, large samples of single W events decaying into $e v_{e}$ and $\mu v_{\mu}$ have been used to measure the $\mathrm{W}$ mass as:

$80.356 \pm 0.125 \mathrm{GeV} / \mathrm{c}^{2}$, an accuracy not sufficient to constrain the Higgs sector. On the other hand, by the end of the LEP 2 run an accuracy of $25 \mathrm{MeV} / \mathrm{c}^{2}$ should be reached. This accuracy will allow the mass of the Higgs to be constrained with a relative uncertainty of $35 \%$ on its mass. Also, since the radiative corrections to the W mass depend on the mass difference between the components of a fermion doublet, $\Delta M_{U, D}$, this measurement will be sensitive to a fourth generation of quarks with $\Delta M_{U, D}>80 \mathrm{GeV} / \mathrm{c}^{2}$. This is quite an interesting number since, for the already known doublets, the mass difference is of the order of a few $\mathrm{MeV} / \mathrm{c}^{2}$ for the up-down doublet, increasing to $3 \mathrm{GeV} / \mathrm{c}^{2}$ for the strange-charm doublet, and $170 \mathrm{GeV} / \mathrm{c}^{2}$ for the top-bottom doublet. Moreover, in the

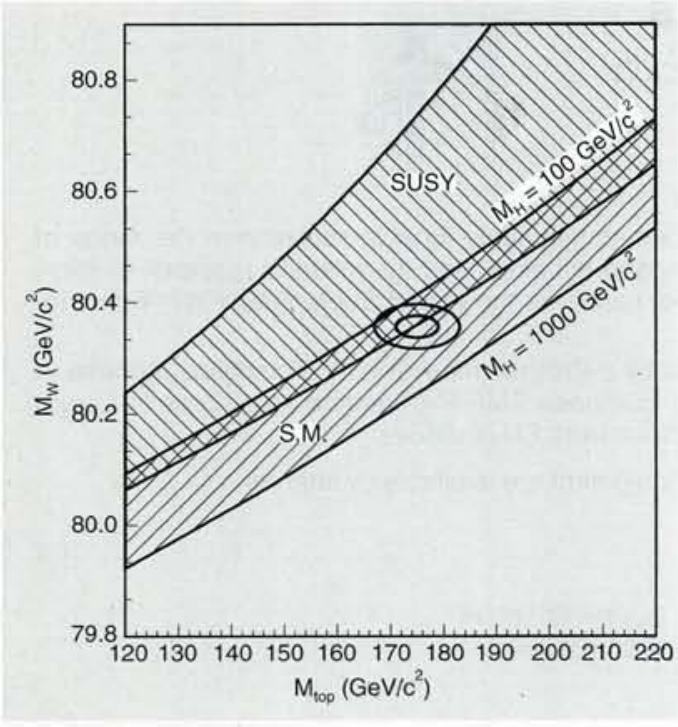

Fig. 1. Relations between $\mathrm{W}$ and top masses "minimal" supersymmetric model with extra corrections due to the supersymmetric partners of the currently known particles, the net radiative correction (at present calculated to first order only) is substantially different from that of the Standard Model [1]. Figure 1 shows, in the $\left(M_{\text {top }}, M_{W}\right)$ plane, the allowed contours with the hypothesis that the direct searches for the Higgs or supersymmetric particles will have failed at the end of LEP, together with the expected uncertainties on the top and W masses. Such accuracy will allow one of the two models to be selected, except in the region of $\mathrm{W}$ and top quark masses where both models overlap. However, this region corresponds to supersymmetric particles with masses greater than $1 \mathrm{TeV}$.

Two techniques for the measurement were foreseen during the workshop on $\mathrm{LEP}_{2}$ physics organized at CERN during the year 1995 [2]:

1) the measurement of the total $e^{+} e^{-}$ $W^{+} W^{-}$cross section at threshold

2) the direct mass measurement above threshold.

These methods were adapted to the time schedule for the increase of the number of superconducting RF cavities. Moreover, they constitute very different systematics since the first consists of counting the number of $\mathrm{W}$ pairs produced, whereas the second is a direct measurement from the reconstructed 4 -vectors of the particles from the decay of the $\mathrm{W}$ bosons.

The accelerator was run at a centre of mass energy of $161 \mathrm{GeV}$ in June-August, and at $172 \mathrm{GeV}$ in October-November 1996. At both energies the ALEPH, DELPHI $\mathrm{L}_{3}$ and OPAL experiments recorded data corresponding to a luminosity of around $10 \mathrm{pb}^{-1}$ each. A preliminary analysis of the data taken at $161 \mathrm{GeV}$ was presented on Ocober 8 by the LEP electroweak working group and is summarized in the following. This energy was chosen because it is just above the threshold for $\mathrm{W}$ pair production, and calculations of the cross section had shown that its slope is maximum at this point, thus providing the optimum sensitivity.

Each W decays into either a leptonantineutrino or a quark-antiquark pair leading to a $l v l v, l v q q$ or 4 jet final state, with respective branching ratios of $1 / 9$, $4 / 9$ and $4 / 9$. Jets are formed of the particles (pions, kaons, etc.) from the hadronization of quarks and gluons.

Typical efficiencies (with reference to background contamination) range from 


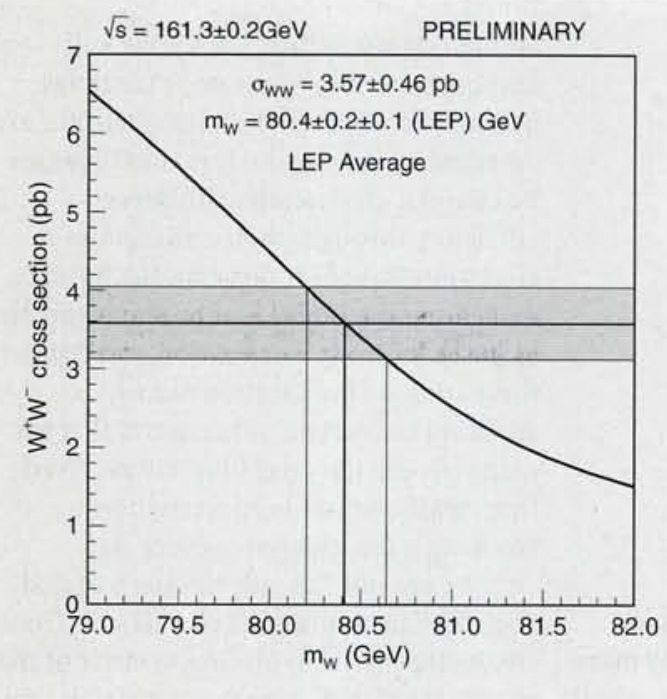

Fig. 2. Cross sections as a function of mass

$60 \%(3 \%)$ in the purely leptonic channel, to $75 \%(7 \%)$ in the semi-leptonic channel, and $85 \%(25 \%)$ in the purely hadronic channel. The easiest channel to detect is lvqq using isolation and lepton identification. In the hadronic channel, multivariable analyses were performed because a simple selection would lead to too low a signal/background ratio.

Such techniques outperformed the results from the LEP2 workshop and led, after combining the results from the four experiments, to a measured cross section of: $3.57 \pm 0.46 \mathrm{pb}$. This result is then compared with the theoretical curve (see Fig.2) which relates the production cross section to the mass. The main systematic uncertainty comes from the knowledge of the beam energy. At present, specialists of the machine quote a $100 \mathrm{MeV}$ uncertainty. The above measurement then translates into: $M_{W}=80.4 \pm 0.2 \pm 0.1$ (LEP) $\mathrm{GeV} / \mathrm{c}^{2}$.
The detailed analysis of the beam energy calibration data will hopefully soon achieve an accuracy expected to lie in the $15 \mathrm{MeV}$ range. Such a result is very encouraging in view of an accurate measurement of the $\mathrm{W}$ mass.

Indeed, a very first look at the $172 \mathrm{GeV}$ data shows that a similar or even better accuracy can be obtained with the direct reconstruction method, and with the total statistics accumulated overall last year at LEP by the four experiments $\left(80 \mathrm{pb}^{-1}\right)$, we should already reach an uncertainty for the mass of the $\mathrm{W}$ of about $125 \mathrm{MeV} / \mathrm{c}^{2}$, a value competitive with the Fermilab result established under very different conditions.

In the coming years of running at higher energy, the uncertainty should reach the projected value $25 \mathrm{MeV} / \mathrm{c}^{2}$ by the completion of the $\mathrm{LEP}_{2}$ programme in 1999, thus performing an unprecedented test of the Standard Model, and perhaps we shall see the indirect manifestations of new physics.

\section{References}

[1] P.H. Chankowski et al., Nucl. Phys. B417 (1994) 101

[2] CERN-PPE/96-01

\section{Press Release}

\section{CERN finds external funding for new antimatter project}

CERN is to build a new experimental facility, the Antiproton Decelerator (AD), by transforming an existing CERN machine, the "Antiproton Collector" which produces and stores antiprotons, into an "all-inone" machine which can additionally decelerate, cool and eject antiprotons at low energies $(5.8 \mathrm{MeV})$. The transformation will cost about 7 million Swiss Francs and will be funded by special contributions from several countries, among them Denmark, Germany, Italy, Japan, Poland and the United States.

The main goal of the new facility is to allow a community of about 150 physicists to continue working with ultra low energy antiprotons, particularly for antihydrogen research. Antihydrogen is an atom made entirely of antiparticles: whilst ordinary hydrogen is a positive proton with a negative electron orbiting around it, the antihydrogen atom has a negative "antiproton" in the centre bound to a positive "positron".

Pioneering experiments at CERN and more recently at Fermilab in the US, had only one aim: to produce and detect a few antihydrogen atoms. They succeeded in demonstrating that antihydrogen can indeed be produced, but the real goal is more challenging: the precise comparison of the physical properties of matter and antimatter atoms. Three-quarters of our universe is hydrogen and much of our knowledge of it has been gained from studying ordinary hydrogen. If the behaviour of antihydrogen differs even in the smallest detail from that of ordinary hydrogen, physicists would have to rethink or abandon many of the established ideas on the symmetry of matter and antimatter.

The answer lies in the study of the antihydrogen atom, best done at rest. The techniques needed to capture antimatter in electrical and magnetic bottles or traps, allowing for high-precision analysis are under intense development at CERN. Two experimental collaborations, ATHENA and ATRAP have been formed to produce antihydrogen and to study its spectroscopy once the AD is in operation, as early as Spring 1999. Each experiment envisages the production and capture of more than 1000 antihydrogen atoms per hour. Antihydrogen promises to be a tool of fantastic sensitivity to find out whether a world made of antimatter would really be indistinguishable from our own.

A third pillar of the AD programme will be a JapaneseEuropean project to investigate the recent discovery that particles of the antiworld are sometimes able to survive ten million times longer than was thought possible, in what is for them a hostile environment that of our everyday world of matter. A general purpose area will also be set up to accommodate a number of small experiments in rapid succession. Monbusho, the Japanese Ministry of Education, Science, Sports and Culture, has announced that the "Antimatter Science" project has been selected as one of the two most important research projects to be carried out from 1998 and will support Japanese participation in the experiments on the $A D$ with funding of approximately 10 million Swiss Francs.

Just over a year after the announcement of the production of the first 9 anti-atoms at CERN in January 1995 , the approval of the AD opens up new exciting research possibilities for scientists from all over the world waiting to increase our knowledge of antimatter. The questions are fundamental: Why, if the same quantities of matter and antimatter were produced during the Big Bang, as is supposed, is our Universe made entirely of matter? Does gravity have the same effect on antimatter as it does on matter? The solutions to these questions could lie in the results which the $A D$ may yield.

CERN, Geneva 